\title{
Agents of Opportunistic Infection
}

National Cancer Institute

\section{Source}

National Cancer Institute. Agents of Opportunistic Infection. NCI Thesaurus. Code C14355.

Infectious agents, which while normally present but remaining benign under the control of the immune system, become virulent and pathogenic in immune-compromised hosts (i.e when they get the opportunity to become infectious). 